Five papers extracted from the reviews identified parental deployment as having a negative effect on school attainment.

Nine studies extracted from the review papers found a positive correlation between having a deployed parent and a greater chance of experiencing depressive symptoms and feelings of anxiety.

Strong correlations of increased prevalence of both externalising \& internalising behaviours were conclusively found in 7 of the reviews.

Increased resilience was detailed in only one study featured in multiple reviews.

Just one study featured across the reviews reported on physiological measures - adolescents with deployed parents had higher blood pressures and significantly higher heart rates and stress scores than civilian children.

Conclusion. More research obtaining the viewpoint of the child directly and observation of such children is required to properly understand the effects on children with a deployed parent, without the interference of parent or teacher reporting bias. Additionally, with only one study reporting on increased offspring resilience there has been limited exploration of potential positive correlates, so further research regarding these is important.

Landscape of childhood and adolescent depression in Pakistan: experience from a tertiary care hospital in Karachi, Pakistan

Maria Khan*, Mohummad Hassan Raza Raja, Fatima Gauhar and Tania Nadeem

The Aga Khan University Hospital

${ }^{\star}$ Corresponding author.

doi: 10.1192/bjo.2021.703

Aims. Depression is highly prevalent in children and adolescents in Pakistan, yet, factors affecting depression have not been widely studied. This study aims to assess the demographic and clinical characteristics of depression in children and adolescents and identify associations between parental marital status and confounding factors for depression.

Method. A descriptive retrospective study was undertaken at the Aga Khan University Hospital in Karachi, Pakistan. Patient records of children and adolescents (aged under 18 years), presenting to the psychiatry clinic with depression from 2015-2019 were reviewed. The diagnosis of clinical depression was made based on clinical assessment according to international guidelines. Patients whose medical records had missing information were excluded. Data were analysed using IBM SPSS Statistics for Windows, version 23.0 (IBM Corp., Armonk, N.Y., USA). Continuous data are presented as mean +/- standard deviation, whereas categorical data are presented as percentages (\%). Pearson Chi-square test of association has been used to assess the association between parental factors and confounding factors. In instances where Pearson's Chi-square test could not be applied, Fisher's exact test is used instead. Associations at p $<0.05$ (95\% confidence limit) are considered statistically significant.

Result. A total of 133 participants were included, of which 78 (58.6\%) were female, and $55(41.4 \%)$ were male, with a mean age of 15.5 +/- 2.4 (Range: Ages 4-18). The population had a $50.4 \%$ prevalence of suicidal ideation, $21.1 \%$ of self-harm, $15 \%$ of substance abuse and $14.3 \%$ of suicide attempts. Academic stress (54.9\%), interparental conflict (30.1\%) and child abuse (29.3\%) were the most common confounding factors reported. Other confounding factors include a family history of depression (20.3\%), experience of bullying
(16.5\%) witnessing domestic violence (16.5\%), substance abuse (15.0\%) and experiencing sexual abuse (6.0\%). There is a statistically significant association between children having parents with non-intact marriages and experiencing sexual abuse $(p<0.001$, Odds Ratio $(\mathrm{OR})=21.48)$, having a family history of depression $(\mathrm{p}<0.001, \quad \mathrm{OR}=7.04)$, child abuse $(\mathrm{OR}=3.78)$. Children of non-traditional (not living with both parents) families were more likely to witness domestic violence $(\mathrm{p}<0.001, \mathrm{OR}=4.28)$, have a family history of depression ( $\mathrm{p}<0.001, \mathrm{OR}=3.44)$, abuse substances $(\mathrm{OR}=3.20)$ and experience child abuse $(\mathrm{OR}=2.48)$.

Conclusion. This study identifies factors that may put children at an increased risk of developing depression and performing highrisk behaviours. The findings can help develop better screening programs and counselling for children and adolescents, allowing prevention and ensuring early diagnosis and care.

\section{Survey of remote consultations in psychiatry during the SARS-CoV-2 outbreak}

Nusra Khodabux*, Satheesh Gangadharan, Samuel Tromans and Avinash Hiremath

Leicestershire partnership NHS Trust

${ }^{\star}$ Corresponding author.

doi: 10.1192/bjo.2021.704

Aims. To compare the usage of remote consultations before and after the first wave of the SARS-CoV-2 outbreak and explore mental health workers' views on the usage of telemedicine.

Method. An online questionnaire survey was developed, and disseminated to mental healthcare professionals via e-mail and social media. Quantitative data were analysed using descriptive statistics and qualitative data were analysed using Braun and Clarke's six step procedure for thematic analysis. 1

Result. There were 40 responses from mental healthcare professionals of varying grades from different sub-specialties, predominantly from the UK. Compared to before the SARS-CoV-2 outbreak, there was an increase in usage of telephone $(9(22.5 \%$ to $(29) 72.5 \%)$ and video consultations $(4(10 \%)$ to $17(42.5 \%)$ ). Respondents reported an increase in virtual MDTs (35(87.5\%) during the pandemic, 9(22.5\%) pre-pandemic).

Based on a 5-point Likert scale, the mean technical quality of telephone consultations was 3.56/5 (Range 2-5), with 75\% rating telephone consultations as not being as good as face-to-face consultations. The mean technical quality of video consultations was $3.58 / 5$ (Range 2-5), with 63\% rating video consultations as not being as good as face-to-face consultations. 25 (62.5\%) respondents felt comfortable using telephone consultations during the pandemic, 20(50\%) felt comfortable using video consultations. Recurring themes identified from the qualitative data regarding reasons for the technical quality ratings were: connection issues, poor infrastructure and security concerns.

Nine $(23 \%)$ respondents felt that using video conferencing consultations had a detrimental impact on the mental health of patients while 14(35\%) felt that telephone consultations had a detrimental impact on patients' mental health. Recurring themes for health practitioners' perceived effect of the use of telemedicine on patients' mental heath were the loss of personal touch and reduced patient engagement.

Conclusion. There was a substantial increase in usage of remote consultations during the first wave of the SARS-CoV-2 pandemic among mental healthcare professionals. The results reported in the present study suggest there are numerous barriers to the use of telemedicine in psychiatry, which require future exploration, ideally through interview or ethnographic studies. 